\title{
基于口啶的手性荧光传感器的合成及其对酒石酸识别性能研究
}

\author{
宋 盼 ${ }^{a}$ 王超玉 ${ }^{a}$ 王 鹏 ${ }^{a}$ 刘晓燕 ${ }^{a}$ 徐括喜*,a,b

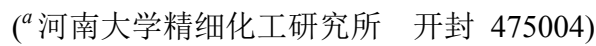 \\ ( ${ }^{b}$ 河南大学环境与分析科学研究所 开封 475004)
}

\begin{abstract}
摘要 以叮啶和 $\alpha$-羟基羧酸为原料合成了三个手性苂光化学传感器 L-1, D-1 和 L-2, 其结构经红外、核磁、质谱表征. 通过紫外光谱、苂光光谱滴定等实验的方法研究了对手性羧酸阴离子的识别能力. 研究结果表明 L-1 和 D-1 具有对酒 石酸阴离子良好的对映选择性识别性能, 且主客体间形成了 $1: 1$ 的络合物.

关键词 呋啶; 手性; 荧光; 化学传感器; 酒石酸
\end{abstract}

\section{Synthesis and Chiral Detecting of Tartrate Fluorescence Sen- sors Based on Acridine}

\author{
Song, Pan ${ }^{a} \quad$ Wang, Chaoyu ${ }^{a} \quad$ Wang, Peng $^{a} \quad$ Liu, Xiaoyan ${ }^{a} \quad \mathrm{Xu}, \mathrm{Kuoxi}^{*, a, b}$ \\ ( ${ }^{a}$ Institute of Fine Chemical and Engineering, Henan University, Kaifeng 475004) \\ ( ${ }^{b}$ Institute of Environmental and Analytical Sciences, Henan University, Kaifeng 475004)
}

\begin{abstract}
Three chiral fluorescence chemosensors L-1, D-1 and L-2 based on acridine were synthesized. The structures of these compounds were characterized by IR, NMR and MS. The chiral recognition property of such sensors was studied by UV-vis and fluorescence titration. The results demonstrate that the sensors L-1 and D-1 exhibit a good enantioselectivity recognition ability toward tartrate and formed $1: 1$ complex.

Keywords acridine; chiral; fluorescence; chemosensor; tartrate
\end{abstract}

叮啶最早是由 Carl Gräbe 和 Heinrich Caro 在 1871 年从煤焦油中提取 ${ }^{[1]}$, 被 Bernthsen 合成制得 ${ }^{[2]} .19$ 世纪 时人们发现活细胞中的核酸能与呋啶衍生物优先结 合 ${ }^{[3]}$, 因而被广泛应用于抗疮药物中，这类物质因为其 药理活性和生物活性吸引了国内外科学家的兴趣. 自此 之后, 有非常多的关于听啶类衍生物在药理和生物活性 方面应用的研究, 如发现呋啶化合物在抗肿瘤 ${ }^{[4]}$ 、抗抗 疮疾、驱虫 ${ }^{[5]}$ 、抗菌 ${ }^{[6]}$ 和治疗艾滋病等方面均表现出很 强的生物活性和药理活性 ${ }^{[7]}$. 目前, 叭啶已经被广泛用 于胺、氨基酸、氨基醇、生物碱和内酰胺抗生素等的合 成 ${ }^{[8]}$. 因呋啶具有良好的发光效应, 苂光性能十分优越, 并且叮啶类的苂光反应体系比较简单, 不需要外加催化 剂, 只要加入到适当的酸碱体系中, 就能发荧光, 而且 发光效率较高, 背景干扰小 ${ }^{[9]}$, 是一类很有前途的苂光 探针, 因此开发性能更优良的苂光探针具有重要意义.
虽然到目前为止, 有关呋啶类的荧光化学传感器已有报 道 ${ }^{[10]}$, 但还鲜有对映选择性识别方面的报道. 在本工作 中, 我们以呋啶和扁桃酸、苯乳酸为原料, 成功地合成 了三个手性荧光化学传感器 L-1, D-1 和 L-2 (Scheme 1), 通核磁氢谱、碳谱、质谱、红外确定其结构, 并通过苂 光光谱等研究了它们对手性羒酸阴离子的识别性能.

\section{1 结果与讨论}

\section{1 苂光光谱研究}

在 D-1 的 DMSO 溶液中分别加入各种常见手性阴 离子, 如酒石酸、扁桃酸、苯乳酸、甲氧基苯乙酸、苹 果酸、二苯甲酰基酒石酸、苯甘氨酸、苯丙氨酸丙氨酸 和谷氨酸等手性阴离子时, D-1 的紫外光谱均未发生明 显的变化. 因此, 我们只使用荧光光谱测量了化合物与

*E-mail: xukx@henu.edu.cn

Received November 4, 2015; revised December 4, 2015; published online December 21, 2015.

Project supported by the National Natural Science Foundation of China (No. U1404207) and the He'nan Innovative Research Team in University (No. 15IRTSTHN005).

国家自然科学基金(No. U1404207)和河南省科技创新团队(No. 15IRTSTHN005)资助项目. 
<smiles>O=S(=O)(O)c1ccc2cc3cccc(CBr)c3nc2c1CBr</smiles><smiles>[R]C(O)C(=O)O[Na]</smiles><smiles>O=C(OCc1cccc2cc3cccc(COC(=O)[C@H](O)[C@H](O)c4ccccc4)c3nc12)C(O)c1ccccc1</smiles>
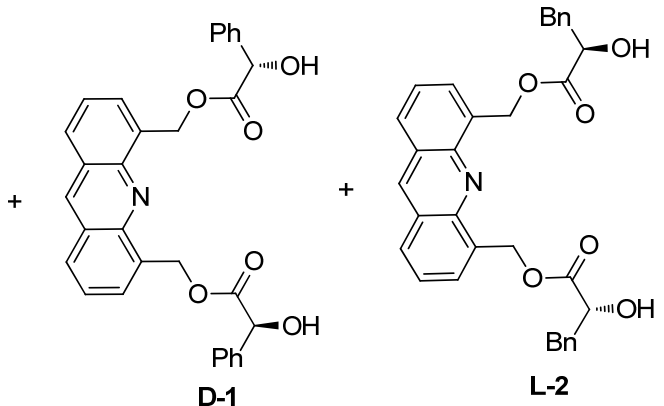

图式 1 传感器 L-1, D-1 和 L-2 的合成

Scheme 1 Synthesis of sensors D-1, L-1 and L-2

底物的相互作用. 图 1 为向 D-1 中加入不同的手性羧酸 阴离子的苂光光谱变化的图, 除酒石酸外, 其他羧酸阴 离子的加入时其荧光光谱的均未发生明显变化.

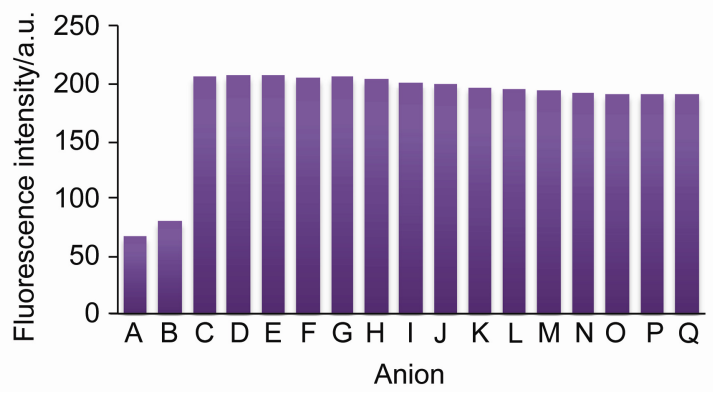

图 1 在 D-1 的 DMSO $\left(3.0 \times 10^{-5} \mathrm{~mol} \cdot \mathrm{L}^{-1}\right)$ 溶液中加入 60 倍 不同阴离子时 $\mathbf{D - 1}$ 的苂光光谱变化 $\left(\lambda_{\mathrm{ex}}=356 \mathrm{~nm}\right)$

Figure 1 Fluorescence spectra changes of sensor D-1 $(3.0 \times$ $\left.10^{-5} \mathrm{~mol} \cdot \mathrm{L}^{-1}\right)$ measured in DMSO upon the addition of 60 equivalent of various anions $\left(\lambda_{\mathrm{ex}}=356 \mathrm{~nm}\right)$

$\mathrm{A}=L$-tartrate, $\mathrm{B}=D$-tartrate, $\mathrm{C}=L$-mandeliate, $\mathrm{D}=D$-mandeliate, $\mathrm{E}=$ $L$-phenyllactiate, $\mathrm{F}=D$-phenyllactiate, $\mathrm{G}=L$-methoxyphenylacetiate, $\mathrm{H}=D$-methoxyphenylacetiate, $\mathrm{I}=L$-malicate, $\mathrm{J}=D$-malicate, $\mathrm{K}=$ $L$-dibenzoyltartrate, $\mathrm{L}=D$-dibenzoyltartrate, $\mathrm{M}=L$-phenylglycine anion, $\mathrm{N}=D$-phenylglycine anion, $\mathrm{O}=L$-phenylalanine, $\mathrm{P}=D$-phenylalanine

为了进一步研究 D-1 对酒石酸阴离子的识别性能, 我们做了苂光光谱滴定实验(图 2,3). 图 $2 \mathrm{~A}$ 和图 $3 \mathrm{~A}$ 分 别是 D-1 与 $L$-酒石酸阴离子和 $D$-酒石酸阴离子作用时 的苂光光谱变化图. 随着酒石酸浓度的增大, D-1 在 430 $\mathrm{nm}$ 处的苂光强度逐渐减弱, 说明 D-1 和酒石酸阴离子 之间发生了相互作用. 苂光猝灭现象可能是由于 D-1 在 与酒石酸阴离子作用过程中, 激发态苂光团听啶和主体 键合单元之间电子转移的自由能 $\left(\Delta G_{\mathrm{PET}}\right)$ 发生变化引起 的. 在 D-1 与酒石酸阴离子发生了配位时其 HOMO 轨 道的电子云密度增加, D-1 键合单元的 HOMO 轨道向激 发态鲐啶的电子转移速率增加, 使得 PET (photoinduced electron-transfer, 光诱导电子转移)过程容易发生 ${ }^{[11 ~ 13], ~}$ 所以观察到 D-1 的苂光猝灭现象. 经最小二乘法非线性 拟合计算得到了它们的络合常数 $(K)$ 分别为 1700 和 933 $\mathrm{L} \cdot \mathrm{mol}^{-1}$, 且相关系数 R 均大于 0.99 , 说明 D-1 与酒石酸
阴离子形成了 $1: 1$ 的络合物(图 $2 \mathrm{~B}$ 和 $3 \mathrm{~B})^{[14 \sim 16]}$.
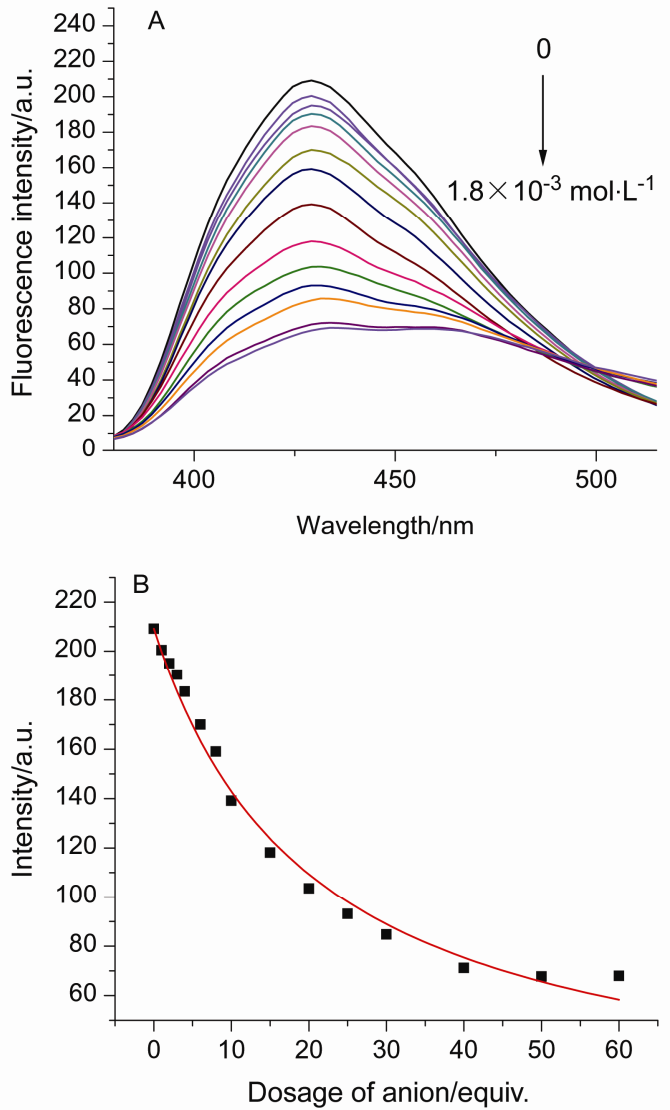

图 2 (A) 在 D-1 的 DMSO $\left(3.0 \times 10^{-5} \mathrm{~mol} \cdot \mathrm{L}^{-1}\right)$ 溶液中加入不 同量 $(0 \sim 60$ 倍于 D-1)的 $L$-酒石酸阴离子时苂光强度的变化和 (B)非线性拟合曲线

Figure 2 (A) Fluorescent intensity changes of sensor D-1 $\left(3.0 \times 10^{-5} \mathrm{~mol} \cdot \mathrm{L}^{-1}\right)$ at $430 \mathrm{~nm}$ with the addition of different amounts $(0 \sim 60$ times of D-1) of $L$-tartrate in DMSO and (B) the non-linear fitting curve

图 4 为向 D-1 中加入 60 equiv.的 $L$-或 $D$-酒石酸阴 离子其荧光变化图, 可以发现 D-1 与 $L$-酒石酸作用时荧 光猝灭的程度大于 $\mathbf{D}-\mathbf{1}$ 与 $D$-酒石酸作用时苂光猝灭的 程度. 说明 D-1 对 $L$-酒石酸的识别能力高于 $D$-酒石酸. 

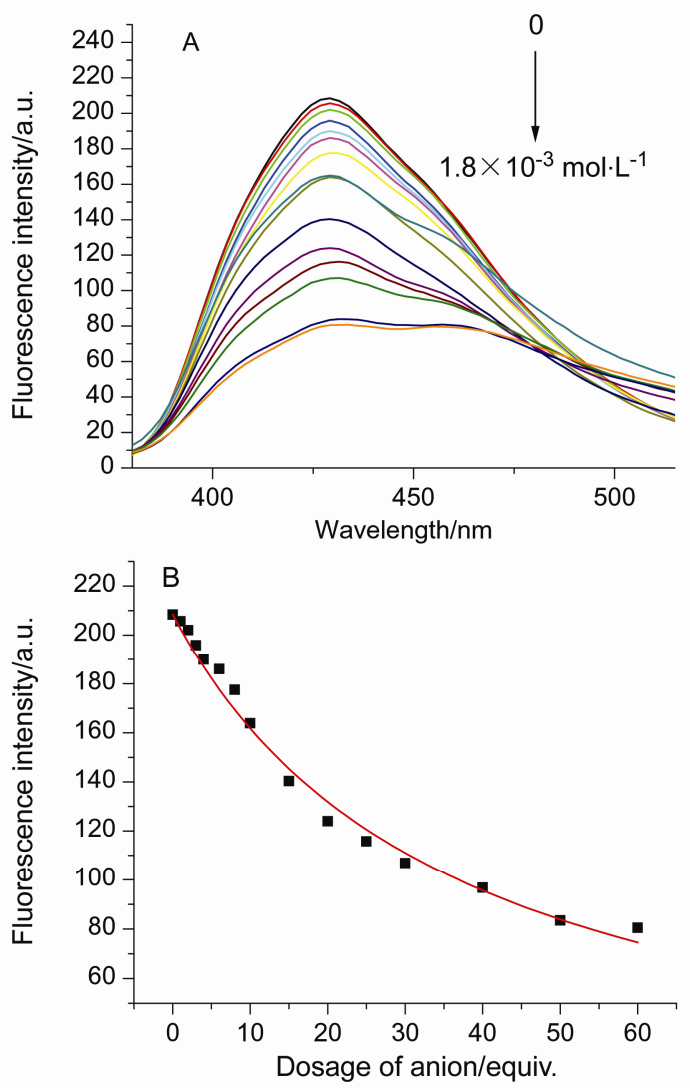

图 3 (A)在 D-1 的 DMSO $\left(3.0 \times 10^{-5} \mathrm{~mol} \cdot \mathrm{L}^{-1}\right)$ 溶液中加入不同 量 $(0 \sim 60$ 倍于 D-1) 的 $D$-酒石酸阴离子时苂光强度的变化和(B) 非线性拟合曲线

Figure 3 (A) Fluorescent intensity changes of sensor D-1 $\left(3.0 \times 10^{-5} \mathrm{~mol} \cdot \mathrm{L}^{-1}\right)$ at $430 \mathrm{~nm}$ with the addition of different amounts $(0 \sim 60$ times of D-1) of $D$-tartrate in DMSO and (B) the non-linear fitting curve

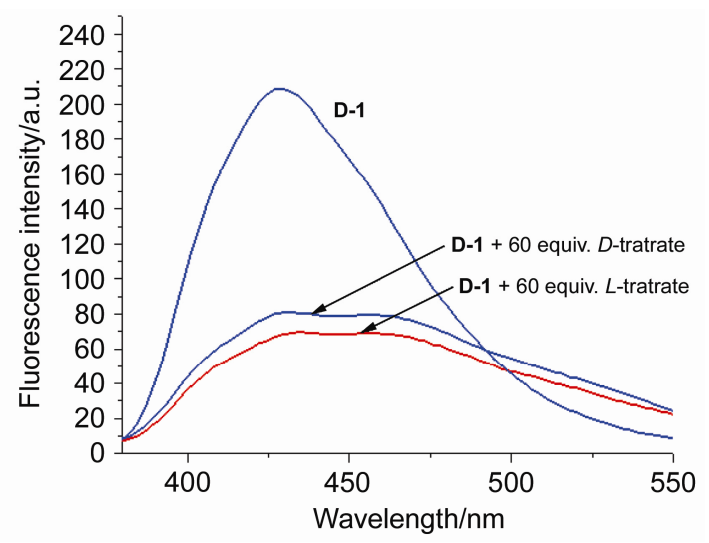

图 4 在 DMSO 溶液中向 D-1 $\left(3.0 \times 10^{-5} \mathrm{~mol} \cdot \mathrm{L}^{-1}\right)$ 中加入 60 倍的 $L-/ D$-酒石酸阴离子时荧光光谱的变化

Figure 4 Fluorescent spectra of sensor D-1 $\left(3.0 \times 10^{-5} \mathrm{~mol}\right.$. $\mathrm{L}^{-1}$ ) with 60 equiv. $D$ - and $L$-tartrate

为了进一步确定主客体相互作用时的化学计量比, 我们测定了其 Job 曲线. 由图 5 可知, 当摩尔分数为 0.5 时, 苂光强度达到最大值, 证明了 D-1 与 $D$ - $/ L$-酒石酸阴
离子均形成 $1: 1$ 的配合物 ${ }^{[16 ~ 18]}$.

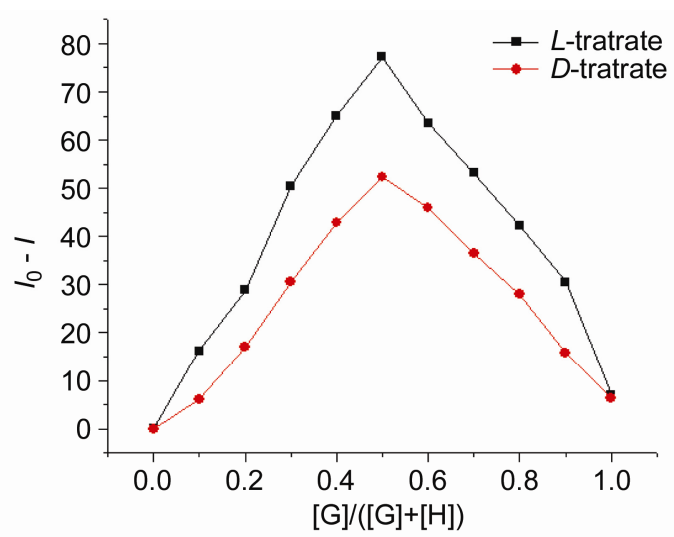

图 $5 \mathrm{D}-1$ 与 $L-/ D$-酒石酸阴离子在 DMSO 溶液中相互作用时 的 Job 曲线

Figure 5 Job plots of sensor D-1 with $D$ - and $L$-tartrate in DMSO The total concentration of the sensor D-1 ([H]) and $D$ - or $L$ - tartrate ([G]) is $\left(3.0 \times 10^{-4} \mathrm{~mol} \cdot \mathrm{L}^{-1}\right)$ in DMSO. $I_{0}$ : fluorescent intensity of sensor $\mathbf{D}-\mathbf{1}$, $I$ : fluorescent intensity in the presence of tartrate

为了篮选出选择性更好的荧光传感器, 我们以苯乳 酸为原料成功地合成了传感器 $\mathbf{L}-2$ 并做了荧光滴定实 验. 研究结果表明, 传感器 $\mathbf{L}-\mathbf{2}$ 与酒石酸阴离子荧光传 感性能相似但络合常数较小, 可能是因为传感器的空间 大小不同和预组织能力的差异致使其对酒石酸阴离子 的识别性能较弱 ${ }^{[15 ~ 18] . ~}$

为了验证D-1 对酒石酸阴离子的对映选择性识别性 能，我们合成了 D-1 的对映体 L-1 并进行了荧光滴定， 并通过最小二乘法非线性拟合计算了其络合常数 $(K)$ 及 相关系数 $(R)$. 表 1 是 D-1, L-1 和 L-2 与酒石酸作用时的 络合常数 $(K)$ 及相关系数 $(R), \mathbf{D}-1$ 对酒石酸的配位平衡常 数比 $\left(K_{L} / K_{D}\right)$ 为 $1.78, \mathbf{L}-1$ 对酒石酸的配位平衡常数比 $\left(K_{L} / K_{D}\right)$ 为 $1 / 1.69$, 说明 D-1 对酒石酸阴离子的识别属于 对映选择性识别.

表 1 L-1, D-1 和 L-2 在 $25{ }^{\circ} \mathrm{C}$ 时与 $D-/ L$-酒石酸盐的络合常数 Table 1 Association constants $\left(K_{\mathrm{s}}\right)$ of sensors L-1, D-1 and L-2 with $D$ - or $L$-tartrate at $25{ }^{\circ} \mathrm{C}$

\begin{tabular}{cccccc}
\hline Entry & Host & Guest & $K_{\mathrm{s}}^{a, b} /\left({\left.\mathrm{L} \cdot \mathrm{mol}^{-1}\right)}^{-} K_{L} / K_{D}\right.$ & $R$ \\
\hline 1 & D-1 & $D$-Tartrate & $955 \pm 3.1$ & \multirow{2}{*}{1.78} & 0.9942 \\
2 & D-1 & $L$-Tartrate & $1700 \pm 3.5$ & & 0.9944 \\
3 & L-1 & $D$-Tartrate & $1096 \pm 1.1$ & \multirow{2}{*}{$1 / 1.69$} & 0.9933 \\
4 & L-1 & $L$-Tartrate & $644 \pm 2.6$ & & 0.9987 \\
5 & L-2 & $D$-Tartrate & $897 \pm 1.4$ & \multirow{2}{*}{0.65} & 0.9967 \\
6 & L-2 & $L$-Tartrarte & $580 \pm 2.8$ & & 0.9950 \\
\hline
\end{tabular}

${ }^{a}$ The data were calculated from the results of the fluorescent titrations in DMSO. ${ }^{b}$ All error values were obtained from nonlinear curve-fitting.

\section{2 核磁共振波谱研究主体 D-1 与酒石酸阴离子的相 互作用}

为进一步了解 D-1 与酒石酸的相互作用细节, 我们 
测定了 D-1 与酒石酸在浓度比为 $1: 1$ 的 DMSO- $d_{6}$ 溶液 中 ${ }^{1} \mathrm{H}$ NMR 谱. 向 D-1 中加入 $D$-或 $L$-酒石酸盐时, $D$-和 $L$-酒石酸盐位于 $\delta 3.66$ 的次甲基 $\left({ }^{*} \mathrm{CH}\right)$ 的共振峰向低场 分别位移了 $\delta 0.12$ 和 0.19 ; 同时 D-1 位于 $\delta 6.02$ 和 5.92 的次甲基 $\left({ }^{*} \mathrm{CH}\right)$ 的共振峰向低场分别位移了 $\delta 0.09,0.10$ 和 $0.13,0.13$, 这说明了 D-1 对 $L$-酒石酸阴离子的络合能 力强于其对 $L$-酒石酸阴离子的络合能力. 这一结果与苂 光滴定的结果是一致的. 在 D-1 与酒石酸盐相互作用时, D-1 位于 $\delta 3.80$ 处的差基的共振峰消失. 上述结果表明, 在与酒石酸阴离子的手性识别过程中, 除了手性匹配作 用外, 还通过了多重氢键的键合作用, 我们推测其可能 的络合方式如图 6 所示.

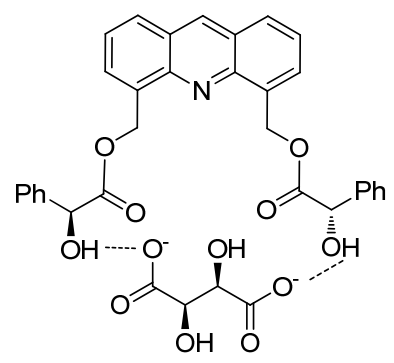

图 6 D-1 与 $L$-酒石酸阴离子的可能络合模式

Figure 6 The possible binding model between sensor D-1 and $L$-tartrate

\section{2 结论}

我们以呋啶和 $\alpha$-羟基羧酸为原料合成了三个手性 苂光化学传感器 L-1, D-1 和 L-2. 并用紫外、荧光光谱 等实验的方法研究了它们对手性羧酸阴离子的识别能 力. 结果表明传感器 D-1 和 L-1 具有对酒石酸盐对映选 择性识别性能且主客体之间均形成了 $1: 1$ 的络合物.

\section{3 实验部分}

\section{1 仪器与试剂}

红外光谱是 Nicolet 670 FT-IR 红外分光光度计测 定, $\mathrm{KBr}$ 压片制样; ${ }^{1} \mathrm{H} \mathrm{NMR}$ 和 ${ }^{13} \mathrm{C} \mathrm{NMR}$ 用布鲁克 $\mathrm{AV}-400$ 测定 (内标为 TMS, 溶剂为 DMSO- $d_{6}$ ); 高分辨 质谱仪型号为 Agilent 1290LC-6540 Accurate Mass Q-TOF(电喷雾电离源). 旋光用 Perkin-Elmer Model 341 旋光仪测定; 紫外光谱是 Hitachi U1900 双光束分光光 度计; 荧光测定用 F-7000 FL 荧光分光光度计; 所用羧 酸阴离子为四丁基铵盐. 4,5-二溴甲基呋啶参考文献 $[19$, 20]上的方法得到.

\section{2 光谱测定}

主客体均配制成 DMSO 溶液. 在光谱测定时, 把主 客体均匀混合, 放置 $2 \mathrm{~h}$ 后测定溶液的紫外或苂光光谱.
将相同浓度 $\left(4.0 \times 10^{-3} \mathrm{~mol} \cdot \mathrm{L}^{-1}\right)$ 的传感器 $\mathbf{L - 1}$ 和 $D$-或 $L-$ 酒石酸阴离子等体积混合后测定 ${ }^{1} \mathrm{H}$ NMR 谱.

\section{3 化合物 L-1, D-1 和 L-2 的合成}

把溶有 $\alpha$-羟基羧酸 $(3.0 \mathrm{mmol}$ )的 $2 \mathrm{~mL} \mathrm{DMF}$ 溶液加 入到含 4,5-二溴甲基鲐定 $(0.37 \mathrm{~g}, 1 \mathrm{mmol})$ 和无水碳酸钾 $(0.41 \mathrm{~g}, 3.0 \mathrm{mmol})$ 的 $25 \mathrm{~mL}$ 无水二氯甲烷中, 完成后加 热回流下反应 $1 \mathrm{~d}, \mathrm{TLC}$ 跟踪. 反应结束后, 加入 $25 \mathrm{~mL}$ 水. 水层用二氯甲烷萃取 $(25 \mathrm{~mL} \times 3)$, 合并的有机相经 盐水洗并用无水 $\mathrm{Mg}_{2} \mathrm{SO}_{4}$ 干燥. 除去溶剂后得到的粗产 品通过硅胶层析柱石油醚/乙酸乙酯 $(V: V=5: 1)$, 得 到浅黄色粘稠固体.

$(S, S)$ - 扁桃酸鲐啶-4,5-二甲酯 $(\mathbf{L}-1): 0.20 \mathrm{~g}$, 产率 $39.4 \%$. $[\alpha]_{\mathrm{D}}^{12}+158\left(c 0.1, \mathrm{CHCl}_{3}\right)$; $(R, R)$-扁桃酸呋啶4,5-二甲酯(D-1): $0.22 \mathrm{~g}$, 产率 $43.3 \%,[\alpha]_{\mathrm{D}}^{12}-155$ (c $\left.0.10, \mathrm{CHCl}_{3}\right) ;{ }^{1} \mathrm{H}$ NMR $\delta: 8.84(\mathrm{~s}, 1 \mathrm{H}), 7.95 \sim 7.89(\mathrm{~m}$, $3 \mathrm{H}), 7.66(J=6.4 \mathrm{~Hz}, 2 \mathrm{H}), 7.60(J=6.4 \mathrm{~Hz}, 2 \mathrm{H}), 7.52$ (s, $1 \mathrm{H}), 7.50(\mathrm{t}, J=8.4 \mathrm{~Hz}, 2 \mathrm{H}), 7.58 \sim 7.42(\mathrm{~m}, 2 \mathrm{H}), 7.40(\mathrm{~d}$, $J=7.6 \mathrm{~Hz}, 2 \mathrm{H}), 7.39 \sim 7.21(\mathrm{~m}, 2 \mathrm{H}), 6.02(\mathrm{~s}, 1 \mathrm{H}), 5.92(\mathrm{~s}$, $1 \mathrm{H}), 5.29 \sim 5.19(\mathrm{~m}, 4 \mathrm{H}), 3.80\left(\mathrm{br}, 2 \mathrm{H}, \mathrm{D}_{2} \mathrm{O}\right.$ exchangeable); ${ }^{13} \mathrm{C}$ NMR $\delta$ : 174.22, 147.51, 146.18, 145.21, 138.58, $138.01,136.89,133.94,133.01,131.99,129.78,129.69$, $128.44,128.79,128.29,127.94,126.71,125.93,125.24$, 71.53, 66.64, 66.21; IR (KBr) v: 3425, 2925, 1738, 1447, $1263,895,756,696 \mathrm{~cm}^{-1}$; HRMS calcd for $\mathrm{C}_{31} \mathrm{H}_{25} \mathrm{NO}_{6} \mathrm{Na}$ $[\mathrm{M}+\mathrm{Na}]^{+}$530.1574, found 530.1577.

$(S, S)$ - 苯乳酸唤啶-4,5-二甲酯 $(\mathbf{L}-2): 0.26 \mathrm{~g}$, 产率 48.5\%. $[\alpha]_{\mathrm{D}}^{12}-23\left(c \quad 0.1, \mathrm{CHCl}_{3}\right) ;{ }^{1} \mathrm{H}$ NMR $\delta: 8.78(\mathrm{~s}$, 1H), $7.98(J=8.4 \mathrm{~Hz}, 2 \mathrm{H}), 7.81$ (d, $J=6.4 \mathrm{~Hz}, 2 \mathrm{H})$, $7.68(\mathrm{~d}, J=6.8 \mathrm{~Hz}, 1 \mathrm{H}), 7.55$ (d, $J=7.2 \mathrm{~Hz}, 1 \mathrm{H}), 7.55 \sim$ $7.51(\mathrm{~m}, 3 \mathrm{H}), 7.17(\mathrm{~s}, 2 \mathrm{H}), 7.17 \sim 7.02(\mathrm{~m}, 5 \mathrm{H}), 5.97(\mathrm{~s}$, $1 \mathrm{H}), 5.89(\mathrm{~s}, 1 \mathrm{H}), 5.66\left(\mathrm{~b}, 2 \mathrm{H}, \mathrm{D}_{2} \mathrm{O}\right.$ exchangeable), 5.36 $5.24(\mathrm{~m}, 4 \mathrm{H}), 4.52 \sim 4.38(\mathrm{~m}, 4 \mathrm{H}) ;{ }^{13} \mathrm{C} \mathrm{NMR} \delta: 174.25$, $147.53,146.21,145.78,138.03,137.25,136.49,133.66$, $132.62,131.68,129.56,129.49,128.76,128.70,128.36$, $128.27,127.81,126.77,126.01,125.62,125.51,71.53$, 65.12, 64.71, 64.17; IR (KBr) v: 3426, 2926, 1738, 1447, 1263, 1177, 895, 757, $697 \mathrm{~cm}^{-1}$; HRMS calcd for $\mathrm{C}_{33} \mathrm{H}_{29} \mathrm{NO}_{6} \mathrm{Na}[\mathrm{M}+\mathrm{Na}]^{+}$558.1887, found 558.1882.

辅助材料(Supporting Information) D-1 与酒石酸阴离 子的紫外工作曲线、核磁滴定图、 L-2 与 $D$-或 $L$-酒石酸 阴离子的苂光滴定图以及各化合物的核磁共振氢谱图. 这些材料可以免费从本刊网站(http://sioc-journal.cn/)上 下载. 


\section{References}

[1] Taner, D. Pure Appl. Chem. 1993, 65, 1319.

[2] Bernthsen, A. Justus, L. Ann. Chem. 1878, 191, 1

[3] Serroni, S.; Campagna, S. Puntoriero, F.; Cinzia, Di P.; McClenaghan, N. D.; Loiseau, F. Chem. Soc. Rev. 2001, 30, 367.

[4] Demonchaux, P.; Laayoun, A.; Demeunynck, M.; Lhomme, J. Tetrahadron 1989, 45, 55.

[5] Bastianelli, C.; Caia, V.; Cumg., Gallo, R.; Mancini, V. ChemInform 1991, 22, 679.

[6] Rbbertson, R. N. Aust. J. Sci. 1945, 7, 112.

[7] Cholewinski, G.; Dzierzbicka, K.; Kolodziejayk, A. M. Pharmacol. Rep. 2011, 63, 305.

[8] Michael, P. D. Asymmetric Catalysis, University of Arizona, New York, 1997, pp. 13.

[9] Sareen, D.; Kaur, P.; Singh, K. Coord. Chem. Rev. 2014, 265, 125.

[10] Jiang, X. J.; Fu, Y.; Xu, L. H.; Lu, H. L.; Zang, S. Q.; Tang, M. S.; Mak, T. C. W. Sens. Actuators B 2014, 202, 388.

[11] Yin, C.; Huo, F.; Zhang, J.; Martínez-Máñez, R.; Yang, Y.; Lv, H.; Li, S. Chem. Soc. Rev. 2013, 42, 6032
[12] Xu, K.-X.; Kong, H.-J.; Li, P.; Yang, L.; Zhang, J.-L.; Wang, C.-J. New J. Chem. 2014, 38, 1004.

[13] Li, Q.; Xu, K.-X.; Song, P.; Dai, Y.-P.; Yang, L.; Pang, X.-B. Dyes Pigm. 2014, 109, 169.

[14] Xu, K.-X.; Kong, H.-J.; Li, Q.; Song, P.; Dai, Y.-P.; Yang, L. Spectrochim. Acta, Part A 2015, 137, 957

[15] Cheng, P.-F.; Jiao, S.-Y.; Xu, K.-X.; Wang, C.-J. J. Org. Chem. 2013, 33, 280 (in Chinese).

(程鹏飞, 焦书燕, 徐括喜, 王超杰, 有机化学, 2013, 33, 280.)

[16] Yang, L.; Xu, K.-X.; Wang, C.-J.; Wang, C.-J. J. Org. Chem. 2013, 33, 2496 (in Chinese). (杨丽, 徐括喜, 王晨娟, 王超杰, 有机化学, 2013, 33, 2496.)

[17] Xu, K.-X.; Xie, X.-M.; Kong, H.-J.; Li, P.; Zhang, J.-L.; Pang, X.-B. Sens. Actuators, B 2014, 201131.

[18] Jian, J.-Y.; Yan, B.-R.; Pan, D.-W.; Tan, Z.; Lü, X.-Y.; Du, H.; Bao, X.-P. J. Org. Chem. 2015, 35, 1069 (in Chinese).

(寒军友, 间柏任, 潘顶伍, 谭赞, 吕新阳, 杜欢, 鲍小平, 有机 化学, 2015, 35, 1069.)

[19] Chiron, J.; Galy, J. P. ChemInform 2004, 14, 2349.

[20] Ebner, C.; Pfaltz, A. Tetrahedron 2011, 67, 10287. 